\title{
Commentary: WHOs Ambition and Action in Nutrition 2016-2025
}

\author{
Wilma B. Freire, PhD
}

\section{Summary}

Margaret Chan, WHO’s outgoing Director-General, introduced the landmark policy statement Ambition and Action in Nutrition 2016-2025 (1) (hereafter, "the document") by stating that "It is unprecedented that nutrition is so high in the political agenda of Member States and the Decade of Action on Nutrition is a unique opportunity to drastically change our food environment, to eradicate hunger and prevent malnutrition worldwide.” Oleg Chestnov, the WHO Assistant Director-General, added that the role of nutrition is an essential component of the 2030 Agenda for Sustainable Development, especially with regard to Sustainable Development Goals 2 (End hunger and achieve food security and promote sustainable agriculture) and 3 (Ensure healthy lives and promote well-being for all at all ages) (1). Thus, WHO explicitly recognizes that malnutrition must be incorporated in its overall agenda, particularly in view of the emerging epidemic of overweight and obesity and their contribution to noncommunicable chronic diseases. More broadly, the links between nutrition, sustainable food systems, and climate change are now increasingly being recognized.

Notably, the document also recognizes the urgent need for improving collaboration within the organization and with other United Nations agencies, especially FAO, UNICEF, and WFP as well as with member states, national public health institutions, academia and research institutions, nongovernmental organizations, the private sector, public/private partnerships, foundations, bilateral development agencies, and local communities.

In this context, the document proposes a vision for the next ten years based on a world free of all forms of malnutrition in which health and wellbeing are achieved by all. Four strategic elements are proposed (1):

- A world: WHO strives for impact in all countries.

- All forms of malnutrition: the double burden of malnutrition must be addressed.

- All people: a life-course approach is essential, including all population groups.

- Health and well-being: WHO's work in nutrition is placed within the wider definition of health.

In order to fulfill its proposed mission to collaborate with member states to achieve universal access to effective nutrition actions and to healthy diets, the document proposes to convene its partners in order to establish, align, and advocate for priority actions to improve nutrition based on available evidence, to support effective implementation activities, and to monitor and evaluate policy and program implementation and nutrition outcomes.

The document defines six priorities across three core functions (leadership, guidance, and monitoring): (i) shape the global nutrition agenda; (ii) leverage changes in relevant non-health sectors to improve nutrition; (iii) leverage the implementation of effective nutrition policies and programs in all settings, including situations of emergencies and crisis; (iv) define healthy diets and guide the identification and use of 
effective nutrition interventions; (v) improve the inclusion of nutrition in health systems; and (vi) support the establishment of targets and monitoring systems in nutrition.

\section{Commentary}

Ambition and Action in Nutrition 2016-2025 represents a substantial proposal that establishes priorities and implementation mechanisms and calls for strengthening WHO's capacity to take corresponding action. It underlines the need to adopt a life cycle focus, and recognizes the role of food systems in contributing to effective solutions. Hence, this document expresses a new WHO strategy and serves as a guide for using its strengths to contribute to solving nutritional problems.

Nevertheless, several questions arise, which should be answered in light of past experience as well as the content of this document:

1. How can WHO assure genuine coordination of efforts directed at specific and measurable objectives within established time frames in a single work proposal that involves different levels within WHO as well as external actors? It is clear that many actors are involved, each of which has its own established objectives and operating procedures. In practice, real problems occur when United Nations agencies try to coordinate among themselves at the international or country levels as well as with governmental agencies. The crux of the problem is that each agency has its own priorities and processes, which are often competitive rather than collaborative and may even come in conflict with other agencies. The same can be said when WHO or other United Nations agencies interact with external actors, including government counterparts.

2. How can WHO put this proposal into effect in a bottom-up fashion, taking advantage of successful experiences at the country and local levels? WHO has years of experience in successfully promoting and supporting the exchange of experiences, effectively involving local professionals to address common objectives. Nevertheless, except in a superficial manner, the document does not propose taking advantage of the value added that local experiences might offer.

3. How can mutually-agreed upon goals be achieved by WHO, both alone and in collaboration with other actors? This is perhaps the greatest challenge because different agendas generally coexist, but they do not necessarily correspond to the common objective of eliminating malnutrition. This is especially so when different actors experience conflicts of interest, as was the case in the debates on tobacco and more recently, with regard to the food and beverage industry and micronutrient producers, whose principal objective is to increase profit. Synchronizing agendas, proposals, programs, and actions represents an enormous challenge, which nevertheless should be prioritized and based from the beginning on a clearly defined operational proposal that allows for evaluating concrete results at the country level. The participation of the private sector in reducing malnutrition can be important, but it is absolutely essential that WHO, in collaboration with other international organizations and member states, take the lead in defining nutritional policies and actions. That is, the response to malnutrition cannot be initiated by Big Tobacco, Big 
Food, or other segments of the transnational private sector. History has shown that transnational corporations have their own priorities, which do not coincide with an evidence-based health agenda or integrated intervention strategies. With their enormous economic power, they can use science (and certain scientists) to achieve their own purposes, thereby even while giving the appearance that they are taking the lead in addressing malnutrition, usually through proposals for simplistic "solutions" to complex problems. There is a vast literature on the insertion of transnational food and beverage corporations in the economies and diets of countries throughout the world. One clear lesson is that a top-down magic bullet approach is generally linked to industrial profit.

4. How can integrated strategies developed by WHO involve actors at the national and local levels with regard to the implementation of priority programs and activities? Experience with vertically-oriented programs shows that they rarely have sustainable impact; nevertheless, they continue to be proposed and implemented in order to address isolated problems, often out of context and without regard to specificities in different countries and cultures. For example, the experience of massive supplementation and fortification programs conclusively demonstrates this point. On the other hand, when local actors actively participate in all stages of interventions, the possibility of sustainable impact is optimized. Without it, lasting results are rarely obtained.

5. What is the role of profit in supplementation and fortification programs? These programs often dominate the international agenda and produce pressure on national governments, usually directly through international agencies that receive and provide substantial economic resources without much regard to detail or even the necessity of those programs (2-6). These simplistic, vertical programs promote specific "solutions" to problems while ignoring the possibility of integrated and potentially more sustainable strategies. This is a challenge that WHO must assume directly and transparently, not only in order to maximize program and policy impact, but also to avoid conflicts of interest, as discussed above (7).

6. What is happening with local capacity building? The document asserts that it is necessary to build professional capacity at the country level. This is an extremely important issue, which should incorporate the latest innovations in knowledge transfer. In the past, WHO has made important contributions in this regard by promoting professional training from the perspective of integrated health services, where the objective was to eliminate malnutrition, strengthen health services, and promote effective local programs. This area has been weakened, though, because imported, one-track proposals have increasingly dominated the scene, and technical personnel and local populations come to play essentially passive roles in receiving and implementing previously designed packages of interventions.

7. What is the role of local universities and research institutions? The contribution of academia outside North America and Europe could be significant were there to be a more proactive approach to their involvement in all stages of program and policy development, evaluation and operations research. In many cases, institutions in Latin America, Africa, and Asia have unique and potentially useful experiences, particularly because they often orient their research and other activities toward local problems and because they represent a useful intersection between government 
agencies and local populations. There are many examples of the critical contributions of local universities, including the schools of public health of Mexico and Sao Paulo. Unfortunately, these and other universities and research institutions were not included in WHO’s Global Nutrition Landscape.

8. What happened to the integrated life cycle approach in nutrition? According to WHO, the problem of malnutrition is present throughout the life cycle, so it is important that the proposed framework include specific actions that include pregnant women, newborns, preschool infants and children, school-age children, adolescents, adults, and older adults, both male and female. Proposals should define specific and integrated interventions regardless of which of WHO's offices or other United Nations agency has operational jurisdiction. A well-designed life cycle focus should result in recommendations and concrete implementation mechanisms. WHO's achievement in breast feeding is an excellent example of what can be accomplished (although in that case, more effective forms of applying the code to the milk substitute industry should be identified). Similarly, proposed activities to be conducted by WHO and other United Nations agencies to promote healthy diets in children, adolescents, and the general population should have common denominators that allow them to reinforce each other and avoid gaps, overlaps, and conflicts. This implies the need for an enormous effort to coordinate activities. For instance, the promotion of healthy diets must be related to the promotion of local food production including, for example, family, school, and community gardens and support for small-scale producers, as well as the consumption of locally-produced foods. Such a integrated orientation would also focus on optimizing food quality, access to a variety of products, and support for the local economy, as well as contributing to limiting the role of the transnational ultra-processed food and beverage industry and even climate change. In this context, the challenge is to develop coordinated programs that combine the specific capabilities of each sector in order to achieve common objectives and results rather than competing among institutions.

Finally, the presence of WHO as the multilateral agency responsible for global health that is both independent and free of conflicts of interest has been and should be the keystone for technical cooperation and support that member states and local communities count on without reservation. 


\section{References}

1. World Health Organization. Ambition and Action in Nutrition 2016-2025. Geneva: World Health Organization, 2017.

2. Gornall J. Sugar's web of influence 2: Biasing the science. BMJ 2015; 350:h215 doi: 10.1136/bmj.h215

3. Chew M, Brizzell C, Abbasi K, Godlee F. Medical journals and industry ties: Zero tolerance on education articles with financial links to industry. BMJ 2014; 349:g7197 doi: 10.1136/bmj.g7197

4. Latham M. The great Vitamin A fiasco. World Nutrition 2010; 1, 1: 12-45.

5. Costello A, Branca F, Rollins $\mathrm{N}$ et al. Health professional associations and industry funding Lancet 2017; 389:597.

6. Thacker P. Coca-Cola's secret influence on medical and science journalists. BMJ 2017; 357:j1638 doi: 10.1136/bmj.j1638.

7. Union of Concerned Scientists. Heads They Win, Tails We Lose: How Corporations Corrupt Science at the Public's Expense. Cambridge, MA: UCS Publications, 2012. 